\title{
Glutathione $S$-transferases as biomarkers of organ damage: applications of rodent and canine GST enzyme immunoassays
}

\author{
Cormac Kilty $^{\mathrm{a}, *}$, Sean Doyle ${ }^{\mathrm{b}}$, Brian Hassett ${ }^{\mathrm{a}}$, Fiona Manning ${ }^{\mathrm{a}}$ \\ a Biotrin, 93 The Rise, Mount Merrion, Dublin, Ireland \\ ${ }^{\mathrm{b}}$ Biology Department, National University of Ireland-Maynooth, Maynooth, Co. Kildare, Ireland
}

\begin{abstract}
The cytosolic glutathione $S$-transferase (GST) enzymes serve as ideal biomarkers of organ damage as they exhibit many of the required characteristics, i.e. specific localisation, high cytosolic concentration and relatively short half-life. The role of GSTs as early indicators of organ damage is applicable to both human and animal models. Because of the regio-specific localisation of the different isoforms of GST in liver and kidney, simultaneous monitoring of classes of GSTs in biological matrices permits the identification of specific areas of damage within a particular organ. Immunoassays have been developed which quantify canine $\alpha$ GST and rodent $\mu \mathrm{GST}$ (Yb1). The immunoassays are solid phase EIAs, where GST in the sample or standard is captured by a specific anti-GST antibody coated onto the solid phase. After washing, a specific enzyme-labelled IgG conjugate is added which binds to the captured GST. After a further washing step, substrate is added and a colour developed. The absorbance is measured on an ELISA plate reader and is directly proportional to the amount of GST present in the sample. The assays are performed at room temperature and can be completed within $3 \mathrm{~h}$. The immunoassays are specific for each GST and have a range of $0-100 \mu \mathrm{g} / \mathrm{l}$. A range of assay parameters were investigated to validate the EIAs for GST detection. The assays are sensitive and reproducible. CV for inter- and intra-assay variation were below $9 \%$ for $\mathrm{Yb} 1$ assay and below $20 \%$ for the canine $\alpha \mathrm{GST}$ EIA. Recovery of spiked GST over the standard curve range was 102 and $99 \%$, respectively. No prozone effect was observed and samples exhibited linearity of dilution in both assays. Validation has shown that using these enzyme immunoassay, Yb1 and canine $\alpha$ GST can be measured accurately
\end{abstract}

\footnotetext{
* Corresponding author.
} 
and precisely in biological matrices, tissue homogenates and cell lines and that changes in GST levels can be detected. The use of these assays have important applications in both in vitro and in vivo toxicity studies, where GSTs serve as sensitive marker of hepatocellular and renal cell integrity. (C) 1998 Elsevier Science Ireland Ltd. All rights reserved.

Keywords: Glutathione S-transferase; Enzyme immunoassay; Toxicity testing; Liver; Kidney

\section{Introduction}

The glutathione $S$-transferases (GSTs) are a family of cytosolic enzymes involved in the detoxification of a range of xenobiotic compounds by conjugation to glutathione. The enzymes consist of homo- and heterodimers grouped into speciesindependent families: alpha $(\alpha)$, pi $(\pi)$, mu $(\mu)$ and theta $(\theta)$ as well as a microsomal trimeric enzyme [1].

In man, $\alpha$-glutathione $S$-transferase $(\alpha$ GST) is the predominant GST isoform in the liver. The uniform hepatic distribution together with relatively high hepatic levels and a short plasma half-life $(\sim 90 \mathrm{~min})$ mean that this enzyme is more sensitive than the aminotransferases as an indicator of hepatocyte status following transplantation, drug-induced liver damage, hepatitis $\mathrm{C}$ infection and $\alpha$-interferon therapy [2-4]. It has recently been reported that monitoring $\alpha$ GST in the first 3 post-operative months after transplantation resulted in improved patient care and reduced mortality and morbidity [5]. Transplant recipients who had $\alpha$ GST reported to the ward, had fewer biopsies, a lower incidence of more severe rejection and fewer episodes of infection. Patients also had shorter stays in hospital post-operatively and better graft survival up to 100 days post-operation.

A similarly unique regio-specific distribution of $\alpha \mathrm{GST}$ and $\pi \mathrm{GST}$ occurs in the kidney. $\alpha$ GST is located primarily in the proximal tubules, whereas $\pi$ GST is present mainly in the distal tubule region of the nephron [6]. A number of different groups have been able to identify specific damage to the proximal or distal tubules in the kidney by monitoring urinary GST. One study looked at kidney damage resulting from the administration of the anaesthetic sevoflurane [7]. The authors were able to distinguish between proximal and distal tubular damage by the simultaneous analysis of urinary $\alpha$ and $\pi \mathrm{GST}$. In another study, psoriasis patients who developed renal impairment while receiving Cyclosporin A (a known nephrotoxin which targets the proximal tubules of the kidney) also showed significantly increased urinary $\alpha$ GST prior to any increase in creatinine or glomular filtration rate (GFR) [8].

Because of the world-wide shortage of organs for transplantation, there is an increasing demand for alternative sources of suitable grafts. Xenotransplantation has been suggested as one possible solution, but many practical as well as ethical problems have been associated with this area. Another option is the use of organs from non-heart beating donors (NHB). $\alpha$ GST has been successfully used to monitor the viability of NHB donor kidneys prior to transplantation [9]. These 
organs are obtained from NHB donors after final and irreversible cardiac arrest and undergo a period of warm ischemia. The duration of this period of ischemia is critical in determining the outcome of transplantation, as increased ischemia times may result in non-viable grafts. Since ischemia predominantly damages cells in the proximal tubules [10], the monitoring of $\alpha$ GST can provide valuable information on the status of the organs prior to transplantation. There was a strong correlation between the release of $\alpha \mathrm{GST}$ and the period of warm ischemia where high levels of $\alpha$ GST were associated with increased warm ischemia times and poor graft function. Thus, by monitoring $\alpha$ GST in the kidney perfusate during machine preservation, the functional outcome of NHB donor grafts can be predicted before transplantation, assisting in the selection of viable organs for transplantation. Levels of $\alpha$ GST can now be used to define reliable safety margins for viability of NHB kidneys.

The main function of the GST family of enzymes is the detoxification of xenobiotics. The protection imparted by these enzymes on the cells against potentially harmful electrophilic compounds is essential in the maintenance of normal healthy processes. However, GSTs have also been implicated in other areas, namely as indicators of malignant transformation and acquired resistance of cells to chemotheraputic drugs [11].

There have been numerous publications on the increased expression of GST, in particular $\pi$ GST, in the malignant transformation of a variety of cells and tissues including bladder [12], head and neck [13], colon [14], ovarian [15,16] and renal cell [17]. Associated with this increased expression of GST is the enhanced detoxification capability of the cell. This has serious implications for drug therapy as acquired resistance can seriously limit the effectiveness of clinically important anti-cancer drugs.

The distribution of GSTs in animal models follows a similar pattern to that in humans and thus they fulfil similar roles as early indicators of organ damage. $\alpha$ GST has been shown to be a more sensitive marker than the transaminases in the assessment of hepatocellular damage in isolated perfused rat livers [18]. In rats, $\mu \mathrm{GST}(\mathrm{Yb} 1)$ has been located to the distal convoluted tubule of the rat kidney, whereas $\alpha \mathrm{GST}$ (YaYc) has been found in the proximal as well as the distal tubule [19].

Compound A, a degradation product of the anaesthetic sevoflurane, was shown to cause an increase in urinary $\alpha$ GST in rats [20]. This increase was mirrored by an increase in proximal tubular cell necrosis, identified by histological examination. The measurement of $\mathrm{Ybl}$ in urine has been shown to be an excellent indicator of nephrotoxicity or kidney damage caused by cisplatin [21]. Other nephrotoxic agents have also been shown to increase kidney Yb1 concentration [22,23].

GSTs have been identified in a number of canine tissues, including liver [24] and lens [25]. As in man, the liver has a high concentration of $\alpha$ GST and canine liver cytosolic GSTs have been purified, characterised and found to contain class $\mu, \alpha$ and $\pi$ subunits, together with a possible $\theta$ class subunit [26]. 
Since enzyme activity assays do not readily facilitate differentiation between the various GST isoenzymes, the development of immunoassays can offer a simple alternative for the specific detection of GSTs. This will allow more accurate monitoring of in vitro or in vivo toxicological studies involving rats or dogs as animal models to predict drug toxicity in humans.

\section{Materials and methods}

\subsection{Purification of GST}

Yb1 GST was purified from rat liver by affinity chromatography according to the method of Hayes [27], with the exception of $S$-hexyl GSH-sepharose being used instead of GSH-sepharose. The procedure involved homogenisation of the tissue, centrifugation and chromatography using $S$-hexyl GSH-sepharose. Purity was established by reducing SDS-PAGE [28] and no significant cross-reactivity was observed with the $\alpha$ or $\pi$ isoforms of rat GST using Western blot analysis [29]. $\alpha$ GST was purified from canine liver obtained from a beagle dog, essentially by GSH-sepharose affinity chromatographic techniques [30]. The liver was homogenised and after centrifugation applied to a GSH affinity column. Purity was established by reducing SDS-PAGE [28]. Using Western blot analysis [29], minimal cross-reactivity was evident for canine $\mu$ or $\pi$ GST isoforms respectively. Protein quantitation was by Coomassie protein assay and also by absorbance measurements at $280 \mathrm{~nm}$.

\subsection{Antibody production and purification}

New Zealand White rabbits were immunised subcutaneously (s.c.) with purified GST, emulsified in Freunds Adjuvant. When the titre was sufficient, the serum was collected and tested for anti-GST specificity by dot blot. IgG present was extracted using Protein A sepharose chromatography. Purified IgG was used for 96-well plate coating and conjugation to horseradish peroxidase, (Yb1 assay) and for conjugation to $N$-hydroxy-succinimido-biotin (canine $\alpha$ GST assay). A murine monoclonal IgG directed against human $\alpha$ GST exhibited $100 \%$ cross-reactivity with canine $\alpha$ GST and was used for 96 -well plate coating.

\section{GST enzyme immunoassay}

Biotrin Canine $\alpha$ GST EIA and Rat Yb1 EIA are quantitative enzyme immunoassays. Both EIA procedures are based on the sequential addition of sample, conjugate and substrate to 96-well plates pre-coated with anti-GST-IgG. The resultant colour intensity is directly proportional to the amount of GST in the sample (Fig. 2). The assay ranges are $0-100 \mu \mathrm{g} / 1$. 


\section{Assay validation}

The following parameters were evaluated in order to validate the EIAs for GST detection: (1) reproducibility (inter- and intra-assay variation); (2) assay sensitivity; (3) parallelism; (4) sample spiking and recovery.

\section{Results}

Antisera raised against both $\mathrm{Yb} 1$ and canine $\alpha$ GST was checked for cross-reactivity with other isoforms of rodent and canine GST respectively by Western blot analysis and EIA. Fig. 1A shows Western blot analysis of Yb1, YaYc and Yp, as well as rat liver and kidney homogenates using anti-Ybl antisera. No significant cross-reactivity was observed with any other major isoform of rodent GST. In the Yb1 immunoassay, no significant cross-reactivity was detected with Yp or YaYc.

Fig. 1B similarly shows the specificity of the monoclonal anti- $\alpha$ GST antibody used for plate coating in the canine $\alpha$ GST EIA. No cross-reactivity was observed

(A) \begin{tabular}{|c|c|}
\hline 1 & Mr Markers \\
\hline 2 & Ybl \\
\hline 3 & YaYc \\
\hline 4 & Yp \\
\hline 5 & Blank \\
\hline 6 & Kidney Homogenate \\
\hline 7 & Liver Homogenate \\
\hline
\end{tabular}

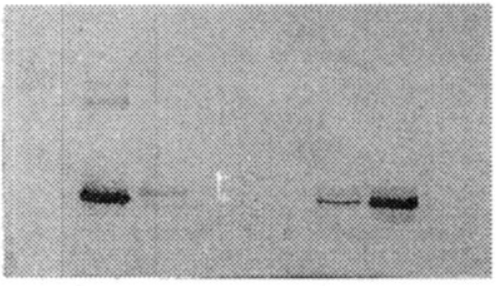

$\begin{array}{lllllll}1 & 2 & 3 & 4 & 5 & 6 & 7\end{array}$

(B)

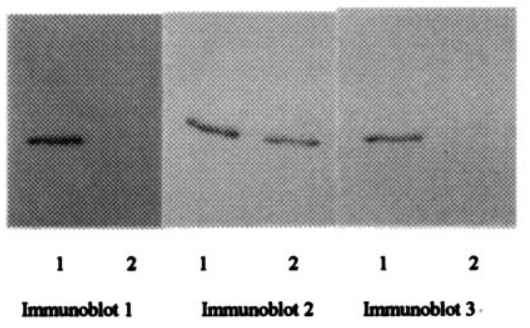

Fig. 1. (A) Immunoblot analysis of rat GST isoenzymes. Evaluation of the specificity of anti-Yb1 antisera used in the Rat Ybl EIA. Tracks 2-4 contain $1 \mu \mathrm{g}$ of GST. (B) Western blots following SDS/PAGE of total canine GST (track 1) and purified Canine $\alpha$ GST (track 2). Immunoblots were probed with monoclonal IgG[anti $\mu \mathrm{GST}]_{\text {human }}$, (blot 1), monoclonal IgG [anti- $\left.\alpha \mathrm{GST}\right]_{\text {human }}$, (blot 2) and polyclonal IgG[anti- $\pi \mathrm{GST}]_{\text {human }}$ (blot 3 ). 


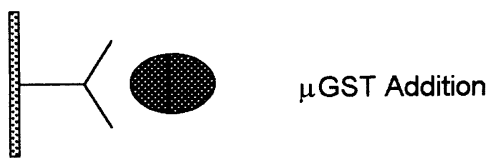

$\lg G$

$\downarrow 60 \mathrm{~min}$
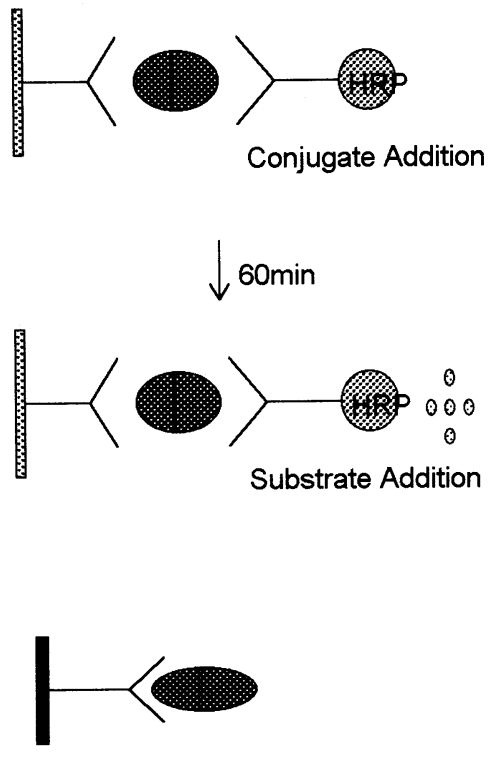

$\alpha$ GST Addition

$\lg \mathbf{G}$

$\downarrow 60 \mathrm{~min}$

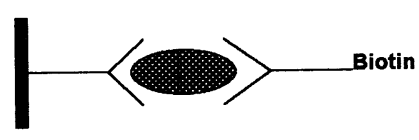

Antibody

Conjugate Addition
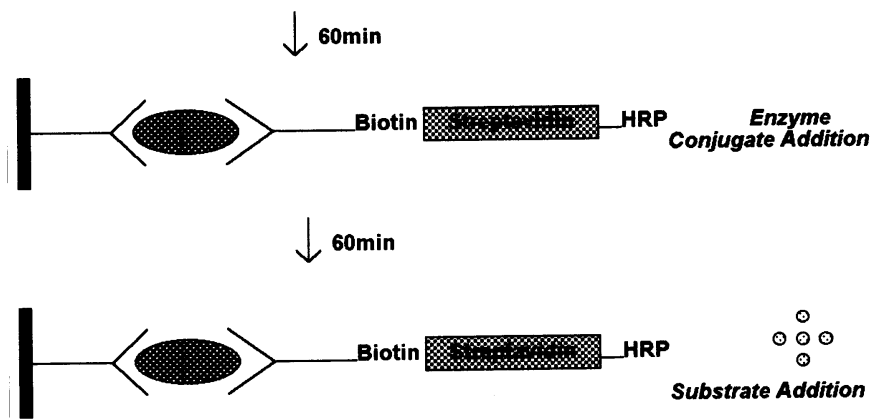

Fig. 2. Schematic diagram of the Rat Yb1 EIA and the canine $\alpha$ GST EIA. 
Table 1

Determination of the sensitivity of the rat Yb1 EIA and the canine $\alpha$ GST EIA

\begin{tabular}{llll}
\hline & Mean O.D. & S.D. & GST concentration $\left(\mu \mathrm{g} / l^{\mathrm{a}}\right.$ \\
\hline Yb1 & 0.125 & 0.006 & 0.232 \\
Canine $\alpha$ GST & 0.152 & 0.085 & 2.094 \\
\hline
\end{tabular}

Sensitivity was determined by assaying the zero calibrator on 30 occasions. The concentration corresponding to the mean O.D. value plus 2 S.D. was deemed to be the limit of detection. The sensitivity of the $\mathrm{Yb} 1$ assay was 0.2 and $2.1 \mu \mathrm{g} / 1$ for the canine $\alpha \mathrm{GST}$ assay in the microwell.

${ }^{\mathrm{a}} \mathrm{A}_{450 / 630} \mathrm{~nm}$ converted to [GST] $\mu \mathrm{g} / 1$ from calibration curve.

with any other isoform of canine GST. In the immunoassay, no significant cross-reactivity was detected with canine $\pi$ or $\mu$.

The assay sensitivity (i.e. the lowest concentration distinguishable from zero) was determined from the analysis of 30 negative samples in the assay. The concentration corresponding to the optical density (O.D.) of the mean of the 30 negatives plus two standard deviations (S.D.) was deemed to be the lower limit of detection of the assay. For the Yb1 EIA, the sensitivity in the microwell was $0.2 \mu \mathrm{g} / 1$, corresponding to $2.0 \mu \mathrm{g} / 1$ in a sample diluted $1 / 10$. In the canine $\alpha$ GST EIA, the sensitivity was determined to be $2.1 \mu \mathrm{g} / 1$ in the microwell, corresponding to an initial sample concentration of $10.5 \mu \mathrm{g} / 1$ when assayed at a $1 / 5$ dilution (Table 1 ).

Precision was investigated as inter- and intra-assay reproducibility. Inter-assay reproducibility was determined based on the analysis of three samples at concentrations spanning the range of the standard curve, assayed in duplicate, in ten separate assays. Intra-assay reproducibility was calculated from the analysis of three samples at varying concentrations, assayed in ten replicates in the same assay.

In the Yb1 assay, the coefficient of variation $(\mathrm{CV})$ for the mean inter-assay reproducibility over the range of the standard curve was $8.5 \%$ (range: $8.3-8.8 \%$ ) (Table $2 \mathrm{~A}$ ), while the $\mathrm{CV}$ for the mean intra-assay reproducibility was $6.2 \%$ (range: 5.1-7.1\%) (Table 2B). In the canine $\alpha$ GST EIA, the CV for the mean inter-assay

Table 2

(A) Inter- and (B) intra- assay variation in the rat Yb1 EIA and the canine $\alpha$ GST EIA

\begin{tabular}{|c|c|c|c|c|c|c|}
\hline \multirow[b]{2}{*}{ A } & \multicolumn{3}{|c|}{ Rat Yb1 EIA } & \multicolumn{3}{|c|}{ Canine $\alpha$ GST EIA } \\
\hline & & & & & & \\
\hline Sample & 1 & 2 & 3 & A & B & $\mathrm{C}$ \\
\hline Mean $(\mu \mathrm{g} / \mathrm{l})$ & 7.4 & 25.1 & 56.1 & 27.8 & 755.7 & 1063.2 \\
\hline S.D. & 0.62 & 2.2 & 4.7 & 5.04 & 47.95 & 64.69 \\
\hline$\% \mathrm{CV}$ & 8.3 & 8.8 & 8.4 & 18.16 & 6.35 & 6.08 \\
\hline \multicolumn{7}{|l|}{ B } \\
\hline Sample & 1 & 2 & 3 & A & $\mathrm{B}$ & $\mathrm{C}$ \\
\hline Mean $(\mu \mathrm{g} / \mathrm{l})$ & 8.3 & 31.4 & 51.2 & 14.8 & 683.6 & 1345.4 \\
\hline S.D. & 0.4 & 2.1 & 3.6 & 3.1 & 55.4 & 133.6 \\
\hline$\% \mathrm{CV}$ & 5.1 & 6.6 & 7.1 & 21.2 & 8.1 & 9.9 \\
\hline
\end{tabular}

Samples were assayed according to the kit instructions. 
Table 3

Sample recovery/linearity of dilution

\begin{tabular}{lll}
\hline Dilution & $(\alpha \mathrm{GST}) \mu \mathrm{g} / 1$ & \\
\cline { 2 - 3 } & Sample diluent & Canine serum \\
\hline $1 / 5$ & $497.41(107.5 \%)$ & $443.61(95.9 \%)$ \\
$1 / 10$ & $484.68(104.8 \%)$ & $472.05(102.0 \%)$ \\
$1 / 20$ & $470.88(101.8 \%)$ & $464.54(100.4 \%)$ \\
$1 / 40$ & $425.72(92.0 \%)$ & $418.32(90.4 \%)$ \\
\hline
\end{tabular}

Canine serum and assay sample diluent were spiked with purified $\alpha$ GST and each assayed at four different dilutions.

reproducibility was $11.5 \%$ (range: $6.7-21.2 \%$ ) (Table $2 \mathrm{~A}$ ), while the CV for the mean intra-assay reproducibility was $9 \%$ (range: $5.1-17.7 \%$ ) (Table $2 \mathrm{~B}$ ).

Tables 3 and 4 illustrate sample recovery/linearity of dilution in the canine GST assay, whereby the recovery of purified $\alpha$ GST spiked into canine serum was compared to that for assay sample diluent. Mean recovery was $99 \%$ (Table 3). Linearity of dilution of canine $\alpha$ GST was observed in other biological matrices including serum, urine and cell culture media (Table 4), confirming the compatibility of these matrices in the immunoassay.

The mean recovery of spiked $\mathrm{Yb} 1$ into rat urine over a range of concentrations from was $102 \%$ (Table 5)

Linearity of dilution for $\mathrm{Yb} 1 \mathrm{was}$ observed in tissue homogenates and urine samples (Table 6) and no prozone effect was observed up to levels of $3.5 \mathrm{~g} / \mathrm{l}$.

The normal range for Yb1 in Sprague Dawley and Wistar rats was established by assaying normal urine samples from 30 Sprague Dawley and 23 Wistar rats. It appears that the normal range of Yb1 differs between different strains of rat. For Sprague Dawley rats, the range was $5-65 \mu \mathrm{g} / \mathrm{l}$, while for Wistar rats a range of $3-30 \mu \mathrm{g} / \mathrm{l}$ was observed. There does not however appear to be any difference between the levels of $\mathrm{Yb} 1$ in urine from female and male rats.

The utility of the canine $\alpha$ GST EIA in measuring hepatic damage has been investigated (Fig. 3). Following warm ischemic damage to the liver in adult beagle dogs, serum $\alpha$ GST levels were measured and compared to the traditional transaminases (GOT and GPT). GST levels showed an initial peak greater than either of

Table 4

Detection and quantitation of canine $\alpha$ GST which was spiked into a variety of matrices including serum, urine and tissue culture medium (M.E.M. and TC-100)

\begin{tabular}{llllll}
\hline Dilution & Sample diluent & Canine serum & M.E.M. & TC-100 & Human urine \\
\hline $1 / 5$ & 400.38 & 348.80 & 382.89 & 365.82 & 433.63 \\
$1 / 10$ & 386.43 & 379.09 & 435.06 & 437.73 & 421.33 \\
$1 / 20$ & 399.10 & 394.90 & 462.40 & 386.96 & 389.32 \\
$1 / 40$ & 435.48 & 440.44 & 416.40 & 376.72 & 406.24 \\
\hline
\end{tabular}


Table 5

Recovery of high and low concentrations of Ybl spiked into samples containing various concentrations of $\mathrm{Ybl}$

\begin{tabular}{lccc}
\hline Sample & Basal level $(\mu \mathrm{g} / \mathrm{l} \mathrm{Yb1)}$ & Final concentration $(\mu \mathrm{g} / \mathrm{l})$ & $\%$ Recovery \\
\hline 1 & 56.41 & 91.83 & 102.7 \\
2 & 106.0 & 141.57 & 104.1 \\
3 & 68.11 & 107.25 & 109.3 \\
4 & 70.78 & 111.76 & 110.9 \\
5 & 17.5 & 115.6 & 98.3 \\
6 & 23.5 & 124.5 & 100.8 \\
7 & 3.2 & 103.3 & 100.1 \\
8 & 15.7 & 105.9 & 91.5 \\
\hline
\end{tabular}

High samples were spiked with $30 \mu \mathrm{g} / \mathrm{Yb} 1$, and low samples were spiked with $100 \mu \mathrm{g} / 1 \mathrm{Yb} 1$. The mean recovery was $102 \%$.

the other enzymes, and also exhibited a more rapid normalisation than the transaminases [31]. This pattern is similar to that observed in studies of liver damage in humans. The canine $\alpha$ GST EIA was also used to quantify $\alpha$ GST as a percentage of total soluble protein in canine liver cytosol $(3.1 \%)$, and in the MDCK cell line $\left(3.87 \mathrm{ng} / 10^{6}\right.$ cells $)$.

\section{Conclusions}

Enzyme immunoassays have been developed for the quantitative determination of canine $\alpha \mathrm{GST}$ and rodent $\mu \mathrm{GST}$ (Yb1) in various biological matrices [31,32]. The assays are performed at room temperature and can be completed within $3 \mathrm{~h}$. Multiple sample analysis is possible. Validation studies have shown that canine $\alpha$ GST and rodent Yb1 can be accurately and precisely determined using the respective enzyme immunoassay and that changes in the levels of GSTs are detectable.

GST Yb1 has been located to the distal convoluted tubule of the rat kidney, whereas YaYc ( $\alpha$ GST) has been found in the proximal as well as the distal tubule [19]. Any event which precipitates distal tubule damage in the rat kidney may cause an increase in urinary $\mathrm{Ybl}$ levels, and a number of nephrotoxic agents have been

Table 6

Linearity of dilution of samples in the rat Yb1 EIA

\begin{tabular}{llll}
\hline Dilution factor & Intestine homogenate $(\mu \mathrm{g} / \mathrm{l})$ & Sample 2 $(\mu \mathrm{g} / \mathrm{l})$ & Sample 3 $(\mu \mathrm{g} / \mathrm{l})$ \\
\hline $1 / 10$ & 222.1 & 606.5 & 115.6 \\
$1 / 20$ & 247.4 & 587.9 & 129.4 \\
$1 / 40$ & 306.0 & 647.9 & 129.6 \\
\hline
\end{tabular}

Samples were serially diluted $1 / 10,1 / 20$ and $1 / 40$ and assayed. 


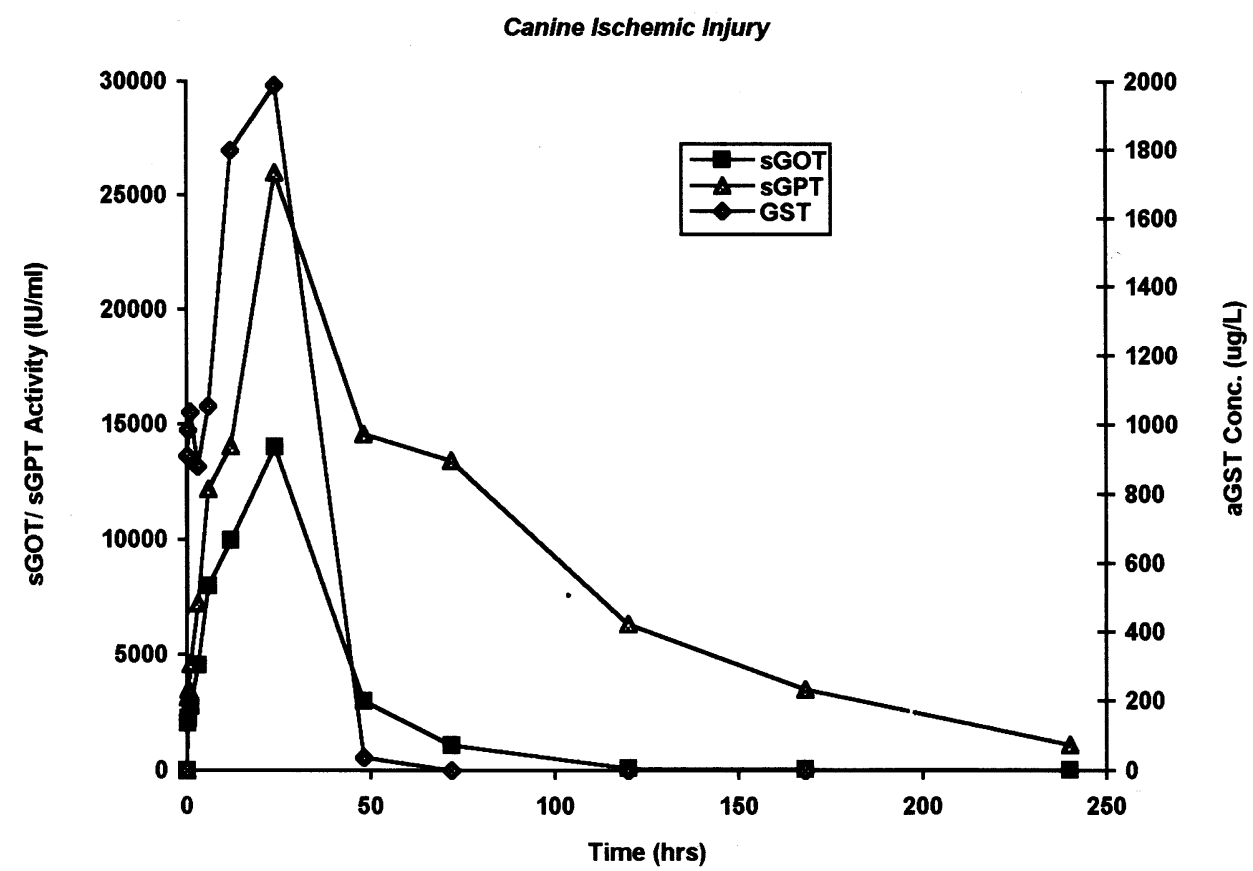

Fig. 3. Serum profile of $\alpha$ GST, sGOT and sGPT in adult female beagle dogs $(8-12.8 \mathrm{~kg})$ following induced warm ischemia by total hepatic vascular exclusion, showing a rapid normalisation of [ $\alpha$ GST].

shown to increase urinary $\mathrm{Yb} 1$ concentrations [22,23]. The measurement of $\mathrm{Yb} 1$ in urine has been predicted as an excellent indicator of nephrotoxicity or kidney damage caused by cisplatin [21]. Thus, by the simultaneous measurement of YaYc and $\mathrm{Yb}$, it may be possible to identify and differentiate between damage to the proximal and distal tubules in the rat kidney.

Elevated levels of canine $\alpha$ GST, present after hepatic injury, are detectable demonstrating the utility of the technique for monitoring in vivo hepatic status. With the concerted efforts to find alternative methods to animal testing for new drugs, the focus is turning to in vitro model systems. The ability to study the effects of novel compounds on primary cells, cell lines or tissue slices grown in static or perfusion cultures will play a pivotal role in these new testing methods. Hepatocytes and renal cells in particular will form the basis of studying the hepato- and nephro-toxic properties of new pharmaceuticals. Because of the specific localisation of GSTs in both of these cell types, the monitoring of GSTs will provide critical information on the status of the cells in any culture system. Indeed studies have already shown that GSTs can be used as markers of cell viability of human liver and kidney slices in an in vitro system, as well as markers for drug-induced toxicity $[33,34]$.

Another important implication of these tests is the ability to distinguish between organ damage in two inter-connected animal model systems. Because of the high 
level of specificity of the rodent and porcine EIAs, no cross-reactivity was observed between the same class of GSTs from different animal species. For example, bioartificial livers (BAL) containing isolated porcine hepatocytes were connected to rats with liver ischemia [35]. By simultaneously monitoring plasma for rodent and porcine $\alpha$ GST, it was possible to differentiate between damage caused to the rodent liver and to the porcine hepatocytes in the BAL. While rodent $\alpha$ GST levels peaked at $6 \mathrm{~h}$ of liver ischemia and gradually decreased at $14 \mathrm{~h}$, no increase in porcine $\alpha$ GST was detected during this time. It was therefore possible to determine that the hepatocytes in the BAL were not effected by the damage caused to the rodent liver and the BAL was capable of maintaining pig hepatocyte integrity in vivo over a period of $14 \mathrm{~h}$.

The necessity of animal toxicity testing of new therapeutics and drugs requires the availability of validated, reliable, easy to use and sensitive tests. GSTs have proved to be sensitive markers of organ and tissue damage caused by a wide range of harmful substances. The easy availability of rapid, easy to use, reliable tests to monitor GSTs in a variety of biological matrices, from a range of animal models facilitates the study of both in vivo and in vitro systems.

\section{Acknowledgements}

We would like to thank Dr Satoru Todo of the Pittsburgh Transplantation Institute, University of Pittsburgh, PA, USA, for access to clinical samples.

\section{References}

[1] G. Beckett, J. Hayes, Glutathione $S$-transferases: biomedical applications, Adv. Clin. Chem. 30 (1993) 281-380.

[2] A. Trull, S. Facey, G. Rees, D. Wight, G. Noble-Jamieson, C. Joughin, P. Friend, G. Alexander, Serum $\alpha$-glutathione $S$-transferase: a sensitive marker of hepatocellular damage associated with acute liver allograft rejection, Transplantation 58 (12) (1994) 1345-1351.

[3] D. Nelson, L. Herbert, D. Oliver, K. Qian, G. Davis, J. Lau, $\alpha$-Glutathione $S$-transferase as a marker of hepatocellular damage in chronic hepatitis C virus infection, Clin. Chem. 104 (2) (1995) 193-198.

[4] D. Thorburn, G. Bird, E. Spence, R. MacSween, P. Mills, $\alpha$-Glutathione $S$-transferase levels in chronic hepatitis $\mathrm{C}$ infection and the effect of $\alpha$-interferon therapy, Clin. Chim. Acta 253 (1996) 171-180.

[5] V. Hughes, G. Alexander, A. Gimson, P. Friend, N. Jamieson, A. Duncan, D. Wight, A. Trull, Randomised trial to evaluate the clinical benefits of serum (-glutathione S-transferase concentration monitoring following liver transplantation. Transplantation 64 (10) (1997) 1446-1452.

[6] J. Campbell, A. Corrigall, A. Guy, R. Kirch, Immunohistological localisation of $\alpha, \mu$ and $\pi$ class glutathione $S$-transferases in human tissues, Cancer 67 (1991) 1608-1613.

[7] E. Egar, D. Koblin, T. Bowland, P. Ionescu, M. Laster, Z. Fang, D. Gong, J. Sonner, R. Weiskopf, Nephrotoxicity of sevoflurane veruses desflurane anesthesia in volunteers, Anesth. Analg. 84 (1997) 160-168.

[8] B. Kirby, S. Rogers, C. Kilty, A. Watson, Urinary glutathione $S$-transferase as an early marker of renal impairment in psoriasis patients treated with cyclosporin (CsA). Presented at XIVth Int. Congress Nephrol., Sydney, Australia. 1997. 
[9] J. Daemen, A. Oomen, M. Janssen, L. van de Schoot, B. van Kreel, E. Heineman, G. Koostra, Glutathione $S$-transferase as predictor of functional outcome in transplantation of machine-preserved non-heart beating donor kidneys, Transplantation 63 (1) (1997) 89-93.

[10] W. Lieberthal, H. Rennke, K. Sandock, C. Valeri, N. Levinsky, Ischemia in the isolated erythrocyte-perfused rat kidney:protective effect of hypothermia, Renal Physiol. Biochem. 11 (1988) 60-69.

[11] D. Schipper, M. Wagenmans, D. Wagener, W. Peters, Glutathione $S$-transferases and cancer (review), Int. J. Oncol. 10 (1997) 1261-1264.

[12] C. Berendsen, W. Peters, P. Scheffer, A. Bouman, E. Boven, D. Newling, Glutathione $S$-transferase activity and subunit composition in transitional cell cancer and mucosa of the human bladder, Urology 49 (4) (1997) 644-651.

[13] T. Mulder, J. Manni, H. Rorlofs, W. Peters, A. Wiersma, Glutathione $S$-transferases and glutathione in human head and neck cancer, Carcinogenesis 16 (3) (1995) 619-624.

[14] R. Butler, W. Butler, Z. Moraby, M. Fettman, K. Khoo, I. Roberts-Thomson, Glutathione concentrations and glutathione $S$-transferase activity in human colonic neoplasms, J. Gastroenterol. Hepatol. 9 (1) (1994) 60-63.

[15] T. Matsumoto, R. Hayse, J. Kodama, S. Kamimura, M. Yoshinouchi, T. Kudo, Immunohistochemical analysis of glutathione $S$-transferase experssion in ovarian tumors, Eur. J. Obstet. Gynecol. Reprod. Biol. 73 (1997) 171-176.

[16] S. Hamada, M. Kamada, H. Furumoto, T. Hirao, T. Aono, Expression of glutathione $S$-transferase- $\pi$ in human ovarian cancer as an indicator of resistance to chemotherapy, Gynecol. Oncol. 52 (3) (1994) 313-319.

[17] D. Grignon, M. Abdel-Malak, W. Mertens, W. Sakr, R. Shepherd, Glutathione S-transferase expression in renal cell carcinoma: a new marker of differentiation, Modern Pathol. 7 (1994) $186-189$.

[18] B. van Wagensveld, J. Scheepers, W. van Gulik, W. Frederiks, D. Obertop, $\alpha$-Glutathione $S$-transferase - a novel parameter for hepatocellular damage in the isolated perfused rat liver. Presented at the 4th ISOS Congress, Washington DC, 1997.

[19] T. Oberley, A. Friedman, R. Moser, F. Siegel, Effects of lead administration on deveolping rat kidney. II. Functional, morphologic and immunohistochemical studies, Toxicol. Appl. Pharm. 131 (1995) 94-107.

[20] E. Kharasch, D. Thorning, K. Garton, D. Hankins, C. Kilty, Role of renal cysteine conjugate $\beta$-lysate in the mechanism of compound A nephrotocity in rats, Anesthesiology 86 (1) (1997) $160-171$.

[21] Y. Sadzuka, Y. Shimizu, Y. Takino, Role of glutathione $S$-transferases in cisplatin-induced nephrotoxicity in rats, Toxicol. Lett. 70 (1994) 211-222.

[22] R. Moser, T. Oberley, D. Daggett, A. Friedman, J. Johnson, F. Siegel, Effects of lead administration on deveolping rat kidney. I. Glutathione $S$-transferase enzymes, Toxicol. Appl. Pharm. 131 (1995) 85-93.

[23] G. Trakshell, M. Maines, Characterization of glutathione $S$-transferases in rat kidney. Alteration of composition by cis-platinum, Biochem. J. 252 (1988) 127-136.

[24] H. Wiener, Heterogeneity of dog liver glutathione $S$-transferases. Evidence for a unique temperature dependence of the catalytic process, Eur. J. Biochem. 157 (2) (1986) 351-363.

[25] T. Nishinaka, R. Kodaka, H. Nanjo, T. Terada, T. Mizoguchi, T. Nishihara, Purification and characterisation of glutathione $S$-transferase isozymes in dog lens, Int. J. Biochem. 24 (11) (1992) $1737-1742$.

[26] T. Igarashi, A. Kohara, Y. Shikata, F. Sagami, J. Sonoda, T. Horie, T. Satoh, The unique feature of dog liver cytosolic glutathione $S$-transferases. An enzyme not retained on the affinity column has the highest activty towards 1,2-dichloro-4-nitrobenzene, J. Biol. Chem. 266 (32) (1991) 2170921717.

[27] J.D. Hayes, Selective elution of rodent glutathione $S$-transferases and glyoxalase 1 from the $S$-hexylglutathione-sepharose affinity matrix, Biochem. J. 255 (1988) 913-922.

[28] U. Laemmli, Cleavage of structural proteins during the assembly of the head of bacteriophage T4, Nature 227 (1970) 680-685.

[29] H. Towbin, T. Staehelin, J. Gordon, Proc. Natl. Acad. Sci. USA 76 (1979) 4350-4354. 
[30] M. Warholm, C. Guthenberg, B. Mannervick, Biochemistry 22 (1983) 3610-3617.

[31] B. Hassett, P. Jennings, S. Doyle, C. Kilty, An enzyme immunoassay for the detection of canine $\alpha$-glutathione $S$-transferase. Presented at the Int. Conf. Glutathione and Glutathione-linked Enzymes in Human Cancer and other Diseases, Hilton Head, USA, 1996.

[32] F. Manning, S. Doyle, C. Kilty, Measurement and quantitation of $\mu$ glutathione $S$-transferase in rat urine by enzyme immunoassay. Presented at the Int. Conf. Glutathione and Glutathione-linked Enzymes in Human Cancer and other Diseases, Hilton Head, USA. 1996.

[33] A. Vickers, Use of human organ slices to evaluate the biotransformation and drug-induced side-effects of pharmaceuticals, Cell. Biol. Toxicol. 10 (5) (1994) 407-414.

[34] H. Clarke, D. Egan, M. Heffernan, S. Doyle, C. Byrne, C. Kitly, M. Ryan, $\alpha$-Glutathione $S$-transferase $(\alpha$-GST) release, an early indicator of carbon tetrachloride hepatoxicity in the rat, Hum. Exp. Toxicol. 16 (1997) 154-157.

[35] L. Flendrig, B. Hassett, S. Doyle, A. te Velde, R. Ladiges, R. Chamuleau, $\alpha$-GST in xenogenic bioartificial liver treatment:Discrimination between recipient and bioreactor originated hepatocellular damage. Presented at the Congress of the ISAO, Rhode Island, USA, 1997. 